\title{
Research on Hydrodynamic Noise of Multistage CentrifugalPump
}

\author{
ZhanqiHao, YuliQian, Haiyan Zhou \\ Water and Power Supply Service Center of North China Oil Field, Renqiu, \\ 062552, China
}

\begin{abstract}
The multistage centrifugal pump is mainly used for urban water supply and residential water, which not only achieves excellent hydraulic performance, but also has higher requirements for noise reduction. This paper focuses on the calculation of the hydrodynamic noise of multistage centrifugal pump, including the calculation of flow field and the calculation of sound field. Then, the calculation results were verified experimentally in this paper to provide some references for the relative researchers.
\end{abstract}

Keywords:hydrodynamic noise, multistage centrifugal pump, sound field of pump

\section{Introduction}

With the development of computer technology, the design, analysis and simulation can depend on the computer, so as to alleviate a lot of tedious work to solve the calculation and interpretation whichhuman cannotrealized. The pump research has entered a new stage. On the other hand, with the continuous development of economy and technology, people on the pump of the increasingly high demand, has not only satisfied to improve its working performance, the pump noise reduction research by more and more scholars and enterprises concerned. In order to reduce the radiation noise caused by the pipeline system, the pump should be designed with low noise. The premise of low noise pump retrofit design, is to establish the pump structure parameters by theoretical or test methods and operating parameters and characteristics of vibration and noise of the contact. However, a lot of model tests need to be carried out in order to sum up the law, the cost is large, and the research period is long. Therefore, in order to 
carry out the optimization design of low noise pump, it is necessary to develop the relevant calculation methods. Because the pump body structure and hydrodynamic noise mechanism are very complex, it is difficult to establish the numerical calculation method of the hydrodynamic noise. With the development of computational fluid dynamics and computational fluid dynamics, the numerical simulation of hydrodynamic noise has been greatly improved. Literature retrieval and related information shows that the problem of hydrodynamic noise in the pump, single stage centrifugal pumpand axial flow pump has been studied. The most typical is the extraction of the dipole source as a sound source, the hydrodynamic noise computational fluid dynamics and computational. For the multistage centrifugal pump, its structure is relatively complex. There are some relevant references. When multistage centrifugal pump works in the field of building water supply system for city residents, we can strictly control the noise.

\section{Calculation of hydrodynamic noise of multistage centrifugal pump}

\subsection{Calculation of flow field}

The head, shaft power and efficiency are the most concerned and important indexes in the design of pump. It is usually hoped that several design parameters can reach the optimal value at the same time. Impeller and guide vane is an important part of the water pump, the change of its structure will greatly affect the performance of the pump. The impeller is the core component of the water pump, which is composed of a cover plate and an impeller blade, the motor drives the impeller to rotate on the shaft, and the energy is transmitted to the fluid through the impeller. The guide vane is pump flow components are important, its role is to collect the impeller thrown out of liquid, the liquid flow rate decreases, the speed can be transformed into a part of pressure, even the introduction of the next stage impeller or conveying fluid. MH120 type multi-stage centrifugal pump structure is complex, the inlet end cap and the outlet end cap of the water area occupies a large proportion, from the flow field and the sound field calculation needs to consider its influence. The acoustic field calculation the dipole source calculation need to export flow field, and the dipole source information is contained in the impeller and the guide vane, each stage impeller and the guide vane surface pressure pulse power are not the same, so the need for simulation of level four. In order to ensure the accuracy and reliability of the numerical simulation, the calculation will be greatly increased. In addition, the impeller mouth ring will lead to water leakage volume, water pump cavity clearance between the impeller and guide vane formation will lead to disc friction loss, the water area includes not only the impeller and the guide vane inlet area, the region also includes cover and impeller front and rear contact. In order to ensure the accuracy of simulation, it is also needed to consider the area of the water body of the mouth ring and the pump cavity. 
The numerical calculation method is used to calculate the pressure and velocity of a series of discrete points, which is achieved by the method of numerical calculation. The mesh consists of structured grid and unstructured grid, the grid structure according to the flow prediction, the need for computational domain block mesh topology, a long time, the higher the requirements of the operator, has the ability of automatic generation of unstructured grid, classification speed, but the computational domain grid is very fast. Under the usual condition, the structure mesh quality is better than that of the unstructured grid, the convergence precision is high, and the convergence rate is fast. As a result, the internal flow analysis has a great impact on the computational domain, as far as possible to the structure of the grid division. Impeller, water pump, leaf cavity structure is complex, has great influence on the internal flow field, numerical pressure, velocity, turbulent kinetic energy change, is an important area. The internal flow field analysis result, the structure of the mesh and the outlet end cap water body shape is relatively complex, the analysis of the internal flow field is not affected, the use of adaptive unstructured grid to divide.

\subsection{Calculation of sound field}

Acoustic finite element method using differential equations and boundary conditions, the equations will calculate the area designated as a certain number of elements, each element connected by nodes, each node element and pressure relationship is determined by the shape function. In the two-dimensional field, the shape function used for tetrahedral and hexahedral elements, in the threedimensional sound field, commonly used for triangular and quadrilateral elements. The system matrix of acoustic finite element simplifies the numerical calculation, and it is convenient to store and calculate the form of symmetric matrix. The acoustic damping matrix is related to frequency, the stiffness matrix and mass matrix are independent of frequency. It can not only calculate the low frequency band, but also deal with the problem of inhomogeneous medium. However, the shape function of acoustic finite element is low order form, sound field is needed when solving a large number of elements, the numerical calculation of the cost will be increased with the model size and frequency range, and the calculation is only applicable to the finite field sound field. The formulation is shown as:

$$
\frac{1}{c^{2}} \frac{\partial^{2} p^{\prime}}{\partial t^{2}}-\frac{\partial^{2} p^{\prime}}{\partial x_{i}^{2}}=\frac{\partial^{2} T_{i j}}{\partial x_{i} x_{j}}-\frac{\partial}{\partial x_{i}}\left(p_{i j} n_{j} \delta(f)\right)+\frac{\partial}{\partial t}\left(\rho_{0} v_{j} n_{j} \delta(f)\right)
$$

The direct boundary element method of LMSVirtual Labis used to calculate the radiated sound field of the inner surfaceand the dipole source radiated from the surface of the blade in this paper. The calculation of acoustic radiation of the volute surface dipole, that the volute is perfectly rigid, the projection surface acoustic scattering and acoustic volute wall method to meet the speed. The total field is a superposition of sound and sound scattering projection. It is calculated from the inner surface of the volute casing surface pressure pulse interpolation to acoustic model. The dipole source is obtained by Fourier transform. In the calculation, the sound source of the spiral case is taken as the boundary condition, 
and the inlet and outlet are sound absorbing boundary. When calculating the sound field radiated from the surface of the blade, it is also considered that the spiral case is completely rigid. The total sound field is the superposition of the surface of the blade and the scattering of the surface of the spiral case. The blade is cut into several parts, and the time history of the pressure pulsation on the blade is carried out according to the time domain of the integral of the area. We make the blade dipole sound source as the boundary condition, the import and export as the sound absorption boundary, and the other wall surface is calculated as the total reflection wall condition.

\section{Experimental verification of calculation results}

Through the hydrophone signal processing, the admittance rate boundary condition of the pump inlet and outlet is obtained, which is shown in figure 1 . The flow of sound source of the sound output interface scroll information into the obtained acoustic radiation of dipole source and volute obtained. It is known that the influence of the impeller rotating dipole can be neglected.The surface dipole of the spiral case is the main noise source in the pump.

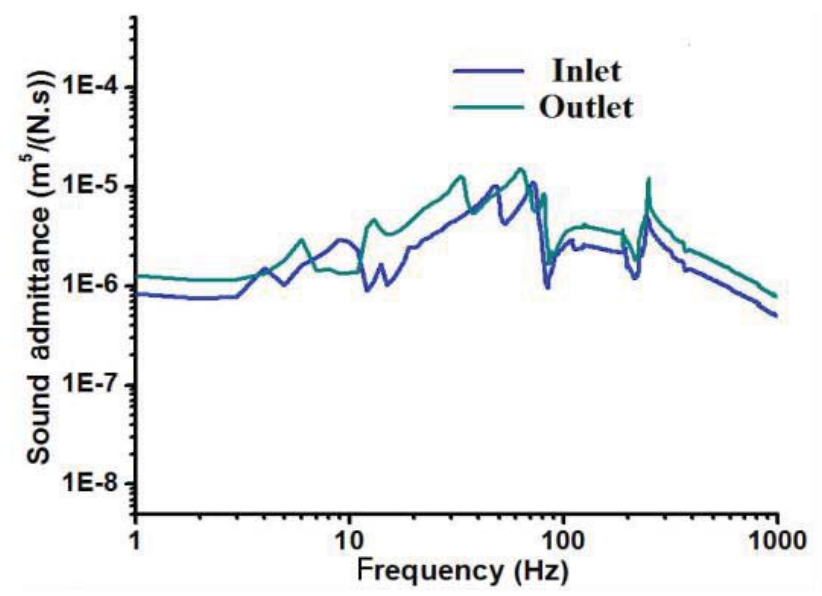

Figure 1 Admittances of inlet and outlet

Pump inlet and outlet sound pressure spectrum is shown in Figure 2. It can be seen from the figure that the frequency of leaf frequency noise is dominant, and the noise level is gradually decreased with the increase of frequency. In addition, the frequency component of each step of the exit is larger than the frequency component of the inlet. The whole pump hydrodynamic noise source has the characteristics of the dipole sound source. 


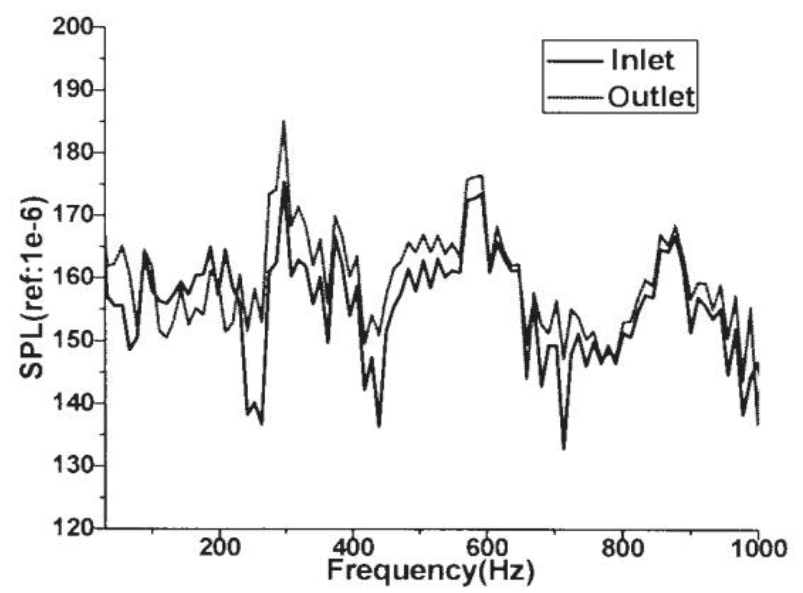

Figure 2 Sound pressure level of inlet and outlet of volute under design working condition

\section{Conclusion}

With the continuous development of science and technology as well as the continuous improvement of material life, people are more and more dependent on the machine. At the same time, the human being has put forward higher requirements for the machinery. Pump is important fluid device. The multistage centrifugal pump is mainly used for urban water supply and residential living water and other occasions. The paper puts forward the basic method to study the hydrodynamic noise calculationof multistage centrifugal pump, which provides a useful reference for the following study.

\section{References}

[1]Ye Xuemin, Pei Jianjun, Li Chunxi, Liu Zi, ExperimentalStudyonNoiseCharacteristicsofCentrifugalPumpBasedonNearfieldAcousticPressure

[2]Method, JournalofChineseSocietyofPowerEngineering, 33(5), 2013.

[3]Zhou Ling, Yang Yang, Wang Wei, Jiang Xiaoping, Shi Weidong, PerformanceandNoiseofMulti-stageCentrifugalPumpAffectedbyImpellerStructure, Water Resources and Power, 34(11), 2016.
[4]Lin
Suqin,
Liu
$\mathrm{Li}$,
Tang
Xiaochen, Modern ResearchProgressonNoiseofCentrifugalPump, IndustrialEconomyandInformationization, 6(21), 2016 